\title{
ELECTRODE BIASING ON TEXTOR: A TOOL FOR FUNDAMENTAL PHYSICS STUDIES
}

\author{
R. R. WEYNANTS,* S. JACHMICH, and M. VAN SCHOOR Laboratory for Plasma Physics \\ Ecole Royale Militaire - Koninklijke Militaire School, Association EURATOM-Belgian State \\ Trilateral Euregio Cluster, B-1000 Brussels, Belgium
}

Received May 3, 2004

Accepted for Publication September 1, 2004

The application in TEXTOR of an externally controlled radial electric field $E_{r}$, imposed by means of an electrode, has allowed to ascertain many aspects of the physics of the creation of $E_{r}$ and of its effect on radial transport. Radial conductivity was shown to depend on parallel viscosity with the latter's nonlinear response to $E_{r}$ providing the basic ingredient for $E_{r}$ bifurcation, typical for $L$ - to H-mode transitions. Simultaneous time and space resolved measurements of $E_{r}$ and of the plasma flows in the edge by means of a newly developed inclined Mach probe have allowed to further substantiate the role of parallel viscosity and of neutral collisions in the damping of rotation. The causal role of grad $E_{r}$ in bringing about the transport changes has been proven by showing that the field shear is spatially correlated with and temporally leads the density gradient, as well as by comparison with theoretical modeling.

KEYWORDS: radial electric field, plasma transport, electrode biasing

\section{INTRODUCTION AND CONTEXT OF THE RESEARCH}

Biasing is the procedure by which a polarized electrode is inserted in the edge of a tokamak plasma with the aim of creating an edge radial electric field $\left(E_{r}\right)$. The importance of radial fields in toroidal plasmas has in the past been repeatedly emphasized. ${ }^{1-6}$ A flurry of activities was prompted by the recognition of a possible role of $E_{r}$ in the establishment of transport barriers either in the edge $^{7-10}$ or more internal to the plasma. ${ }^{11-14}$ This role could be based on the effect of $E_{r}$ or its spatial derivatives on particle orbits ${ }^{15-17}$ that affect collisional ${ }^{18-22}$ and turbulent transport, ${ }^{23-26}$ or, more likely, on the turbulence

\footnotetext{
*E-mail: roger.weynants@ @rma.ac.be
}

level itself. ${ }^{27}$ A reduction of the turbulence can occur either by providing linear stabilization of the turbulent modes $^{28}$ or by achieving nonlinear decorrelation. ${ }^{29-31}$ Bifurcation to improved confinement can be the result of this interaction with turbulence.

Biasing studies in TEXTOR started in 1990, shortly after the pioneering experiments on the CCT tokamak, ${ }^{8,32}$ in which transitions from low to high electric fields could be induced through a process of bifurcation presenting typical signatures of an L-H transition. ${ }^{7}$ Figure 1 shows the usual TEXTOR setup. ${ }^{35}$ The electrode has a canoe- or mushroom-shaped head that is mounted on a steel shaft housed in an insulating sleeve made of boron nitride and inserted 5 to $7 \mathrm{~cm}$ beyond the toroidal belt limiter ALT-II positioned at the geometrical radius of typically $a=46 \mathrm{~cm}$. Please note that the location of the last closed flux surface (LCFS) depends on the actual plasma position. A bias voltage of up to +900 or $-900 \mathrm{~V}$ is applied between the electrode and all eight blades of the ALT-II (all grounded to the liner). Typical machine

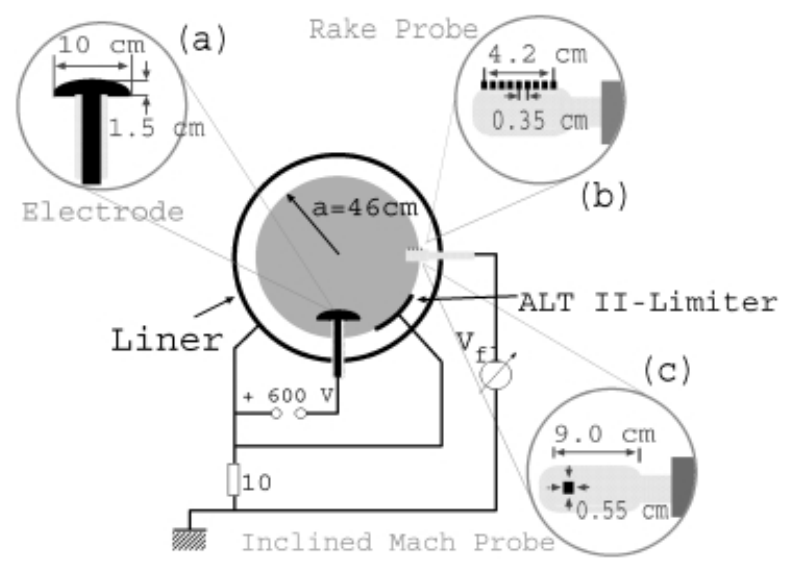

Fig. 1. Poloidal cross section of TEXTOR showing biasing electrode (a) with circuitry and manipulator for the rake probe (b) or the inclined Mach probe (c). ${ }^{33,34}$

FUSION SCIENCE AND TECHNOLOGY VOL. $47 \quad$ FEB. 2005 
characteristics are $B_{\phi, 0}=2.35 \mathrm{~T}, I_{p}=200 \mathrm{kA}$, and $\bar{n}_{e, o}=$ $1-2 \times 10^{13} \mathrm{~cm}^{-3}$. For negative voltages, the electrode current is limited to the ion saturation current, and the absolute value of the ensuing negative radial electric fields is usually much smaller than that obtained with a positive bias. Neutral beam injection has been occasionally used to boost the development of strong negative $E_{r}$, but most results have been achieved with positive biasing. It should be recalled that TEXTOR provided the first firm evidence $^{36}$ that $\mathrm{H}$-mode behavior could be achieved with either polarity of the edge radial field.

When the electrode is biased and an electrode current drawn, the discharge conditions are strongly affected: many plasma parameters change (see Fig. 2 for the time evolution of some of them). The particle and energy confinement is improved. One notes in Fig. 2 the bifurcation in $E_{r}$ (typical for an L- to H-mode transition) and in the electrode current occurring at $t=2.1 \mathrm{~s}$ when a sudden increase of $E_{r}$ is accompanied by a decrease of

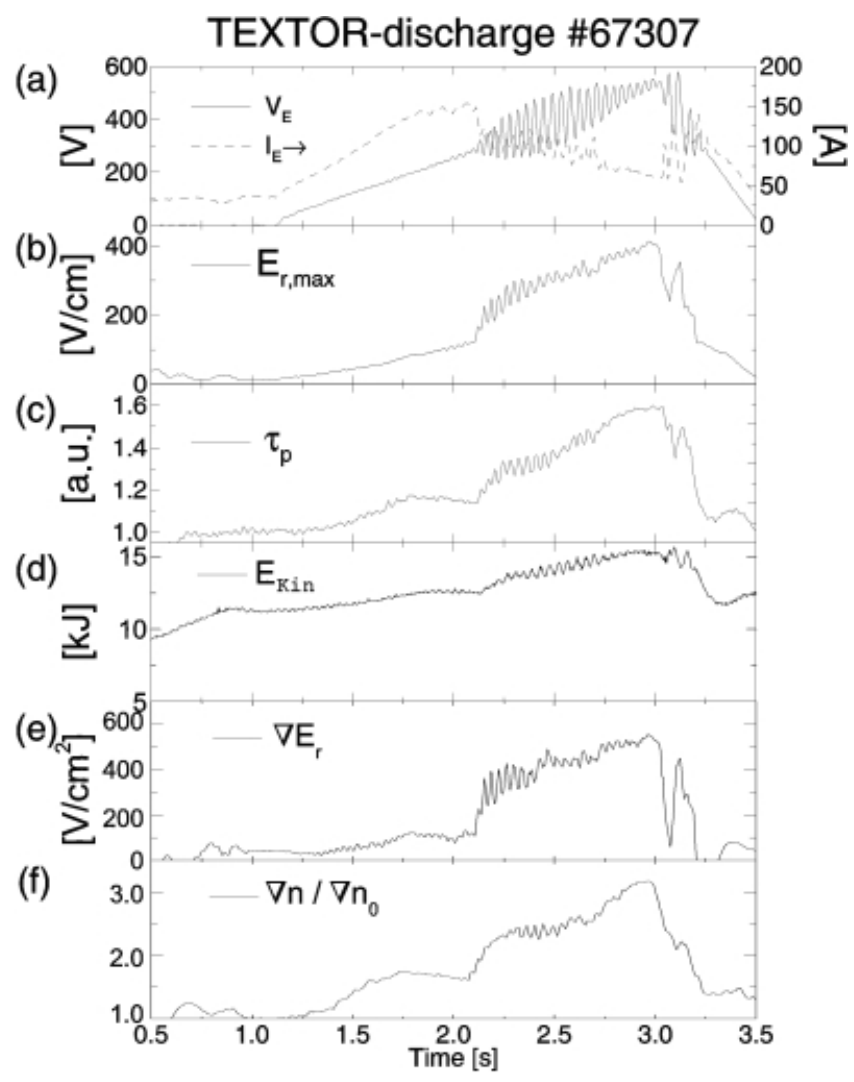

Fig. 2. Temporal evolution of (a) the electrode voltage and current, (b) the maximum electric field, (c) the global particle confinement time, (d) the kinetic energy, (e) the gradient of the electric field and (f) the relative change of the density gradient at the same radial position as (e) $(r=43.9 \mathrm{~cm})$ during biasing. ${ }^{33}$ The spiky behavior on the voltage and some other signals is the result of ringing of the power supply. the current drawn by the electrode. A spatially very narrow and important radial electric field and plasma rotation of appreciable amplitude develop in the edge in the immediate vicinity of the LCFS (Fig. 3) and give rise to a sizable flow shear.

When introducing an electrode in the plasma one can expect that a certain perturbation occurs in the global
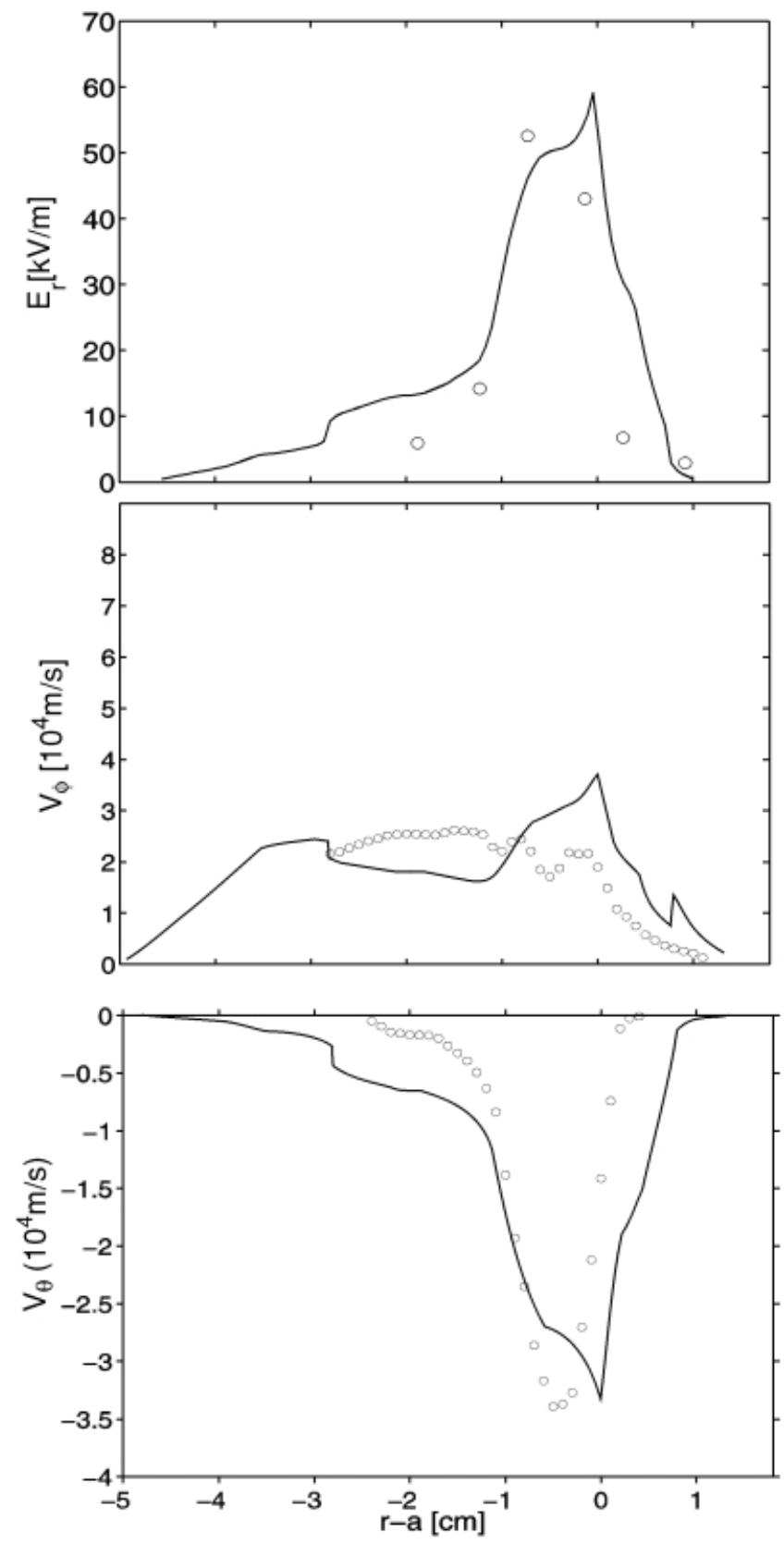

Fig. 3. Typical measured radial profiles (open circles) of the radial electric field $\left(E_{r}\right)$ and the toroidal $\left(V_{\phi}\right)$ and poloidal $\left(V_{\theta}\right)$ velocity and comparison with theory (solid lines $)^{37}$ as a function of the minor radius $[a=r($ LCFS $)$ situated at $r \simeq 46 \mathrm{~cm}$ ]. 
plasma parameters. From the very first experiments, ${ }^{36}$ however, it was found that the introduction of the unbiased electrode has a marginal impact on the loop voltage, the radiated power, and the neutron yield. With biasing the loop voltage essentially remains unchanged and the radiated power and neutron yield increase, in first approximation in agreement with the observed density change. The introduction of the electrode therefore under no condition leads to a substantial impurity increase. On the other hand we wish to mention that detailed studies have been done on the impurity transport with and without bias ${ }^{35,38}$ without being discussed in the current overview.

The biasing experiments on TEXTOR have allowed to carry out many fundamental and original studies on the physics of radial transport, radial conductivity, rotation damping, and turbulence decorrelation and necessitated the development of measurement techniques to assess the parallel as well as the perpendicular rotation in the plasma. All these aspects will be briefly reviewed in this paper. Please note that this is an overview paper, combining results from different research papers, spanning 12 years of research. As a result, the experimental conditions change around the typical values given above, possibly resulting in changes in the ensuing plasma parameters.

\section{STUDIES ON RADIAL CONDUCTIVITY, PARALLEL VISCOSITY, AND NEOCLASSICAL TRANSPORT}

Figure 4 represents the first-ever measured relation between the radial current density and the applied elec-

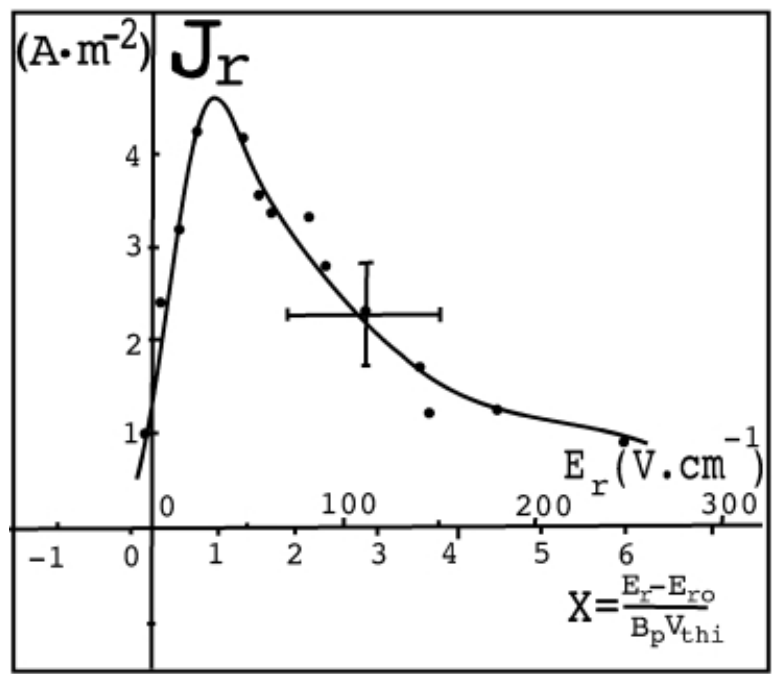

Fig. 4. Radial current density $J_{r}$ versus $E_{r}$ in a tokamak, illustrating how the conductivity of the plasma varies with the applied electric field. tric field, obtained in TEXTOR by means of biasing in 1990 (Ref. 39), and shows how the radial conductivity varies with the electric field. Plotted in Fig. 4 is the measured current density $\left|J_{r}\right|=\left|I_{E} / S\right|$ versus the measured value of $E_{r}$ at the location of its maximum value in the profile (see also Fig. 3) under the prevailing experimental conditions. For $S$, the total area of the closed magnetic surface at the radius where $E_{r}$ is obtained is used. The uniform toroidal distribution of the electrode current is experimentally verified by measuring the equal contributions on each ALT blade, the poloidal symmetry being inferred as a consequence of the existence of rotational transform.

The most salient observation is the fact that there is a maximum current density the plasma can sustain and that the mentioned bifurcation of $E_{r}$ occurs approximately when this maximum is reached.

The experimental results exemplified by Figs. 3 and 4 triggered a spell of theoretical activities both by the TEXTOR team ${ }^{37,40}$ and by others, ${ }^{41-44}$ the contents and findings of which can be summarized as follows:

1. A rather instructive way to relate $J_{r}$ and $E_{r}$ starts from the radial component of Ohm's law, which links, to a very good approximation, ${ }^{45}$ the radial electric field to the toroidal and poloidal rotation velocities of the ions and to the ion pressure gradient according to Eq. (1), where the Spitzer resistivity has been neglected:

$$
E_{r}=V_{\phi, i} B_{\theta}-V_{\theta, i} B_{\phi}+\frac{1}{e_{i} n_{i}} \nabla p_{i} .
$$

The steady-state poloidal $(\theta)$ and toroidal $(\phi)$ components of the ion rotation velocity $\vec{V}_{i}=\vec{V}$ can then be obtained in relation to $J_{r}$ from the single-fluid momentum Eq. (2):

$$
\begin{aligned}
0= & \rho(\vec{V} \cdot \vec{\nabla}) \vec{V}+\vec{M}_{e}+\vec{M}_{i}+\vec{J} \times \vec{B} \\
& -\vec{\nabla}\left(p_{i}+p_{e}\right)-\vec{\nabla} \cdot \Pi_{i}-\vec{\nabla} \cdot \Pi_{e}-\rho \nu_{i o} \vec{V},
\end{aligned}
$$

where $\vec{M}_{\alpha}$ is an external momentum source for species $\alpha=e$ or $i, \Pi_{\alpha}$ is the stress tensor, and $\rho \nu_{i o} \vec{V}$ describes a possible friction force on the ion fluid by the neutrals. Note in passing that a steady-state $J_{r}$ can only exist as a so-called return current, counteracting loss cone losses of particles, ${ }^{9,46}$ collisionless currents of radio frequency or magnetohydrodynamic driven ions or electrons ${ }^{47-49}$ or, as is the case here, an externally imposed biasing current. ${ }^{8,32,36}$ Of crucial importance in the analysis is the parallel component of the ion stress tension term, which, neglecting all anomalous effects and only retaining neoclassical parallel viscosity assumes under the appropriate averaging conditions, ${ }^{50}$ the generic form $\langle\vec{B} \cdot \vec{\nabla} \cdot \overrightarrow{\vec{\pi}}\rangle=C\left(\left\langle V_{\theta}\right\rangle-\right.$ $\left.V_{\text {neo }}\right)$, where $C \approx\left(B_{o} \Theta m_{i} n q V_{t h, i} / R_{o}\right) \exp \left(-X^{2}\right), \Theta$ is the magnetic pitch, $X=\left(E_{r}-E_{r o}\right) / B_{\theta} V_{t h, i}, E_{r o}$ is the ambipolar field, $V_{\text {neo }}=1 /\left(Z e B_{o}\right) \nabla_{r} T_{i},\langle\ldots\rangle$ denotes the flux surface average, and the other symbols have their usual meaning. 
Flux surface averaging with appropriate coefficients of the $\theta$ and // components of Eq. (2) results in a link between $\left\langle V_{\phi}\right\rangle,\left\langle V_{\theta}\right\rangle$, and $\left\langle J_{r}\right\rangle$. With Eq. (1) it is then possible to write the current density as a function of $E_{r}$.

2. The analysis thus shows that the observed radial conductivity can be linked to the damping of poloidal rotation by parallel viscosity, a result already theoretically predicted in the context of neoclassical transport. ${ }^{18,19}$ The destruction of parallel viscosity when $X$ grows larger than unity, on account of the destruction of banana orbits under these conditions, is still today the most plausible explanation for the existence of the maximum current density that occurs around $X=1$ (see Fig. 4). It also provides the essential nonlinearity for bringing about the observed bifurcation in $E_{r}$ (see Fig. 2b).

3. Given the well-known observation that turbulent transport fluxes surpass the neoclassical ones by far, the fact that radial conductivity in a plasma edge would manifest neoclassical features is a priori hard to believe. The experimental evidence therefore appears to show that edge turbulence in TEXTOR under these experimental conditions is of an electrostatic nature, as the then turbulently induced fluxes are expected to be intrinsically ambipolar. $^{51}$

4. More detailed studies have been made on the radial localization of $E_{r}$ (Refs. 40-44) and on the consistency of the field and the underlying flows ${ }^{37}$ An example is shown in Fig. 3.

In addition to upholding the role of parallel viscosity, this modeling identifies neutral collisions as a further important sink of rotation (in fact the main one for toroidal rotation) at the plasma LCFS, where it prevents $E_{r}$

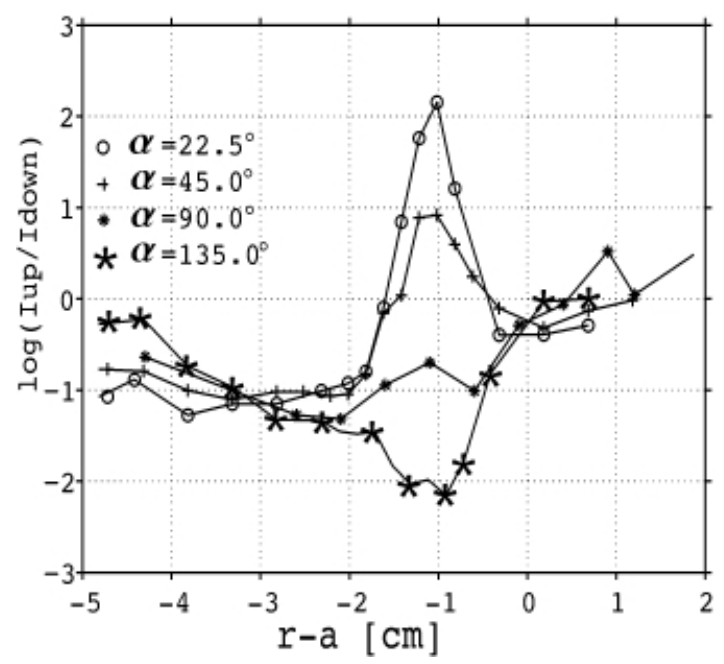

Fig. 5. The ratio of the up- and downstream saturation current as a function of the inclination $(\alpha)$ of the Mach probe's surface with respect to the magnetic field $[a=r$ (LCFS) situated at $r \simeq 48.2 \mathrm{~cm}]$. from growing after the bifurcation. ${ }^{52}$ It further points to the importance of plasma compressibility ${ }^{50}$ at the high rotation speeds typical for these conditions.

\section{EXPERIMENTAL INVESTIGATION OF THE PLASMA ROTATION}

For the in-depth investigation of the role of the electric field on rotation and turbulence quenching in the edge, an accurate measurement of the rotation velocities, parallel as well as perpendicular (but still in the same magnetic surface) to the magnetic field, is indispensable.

Although the measurement of the flow parallel to the magnetic field is well documented, ${ }^{53-55}$ a new method had to be developed to measure the parallel flow as well as the flow perpendicular to the magnetic field. It was shown that by inclining a Mach probe with respect to the toroidal magnetic field, the ratio of the up- and downstream saturation currents reacts strongly to nonparallel
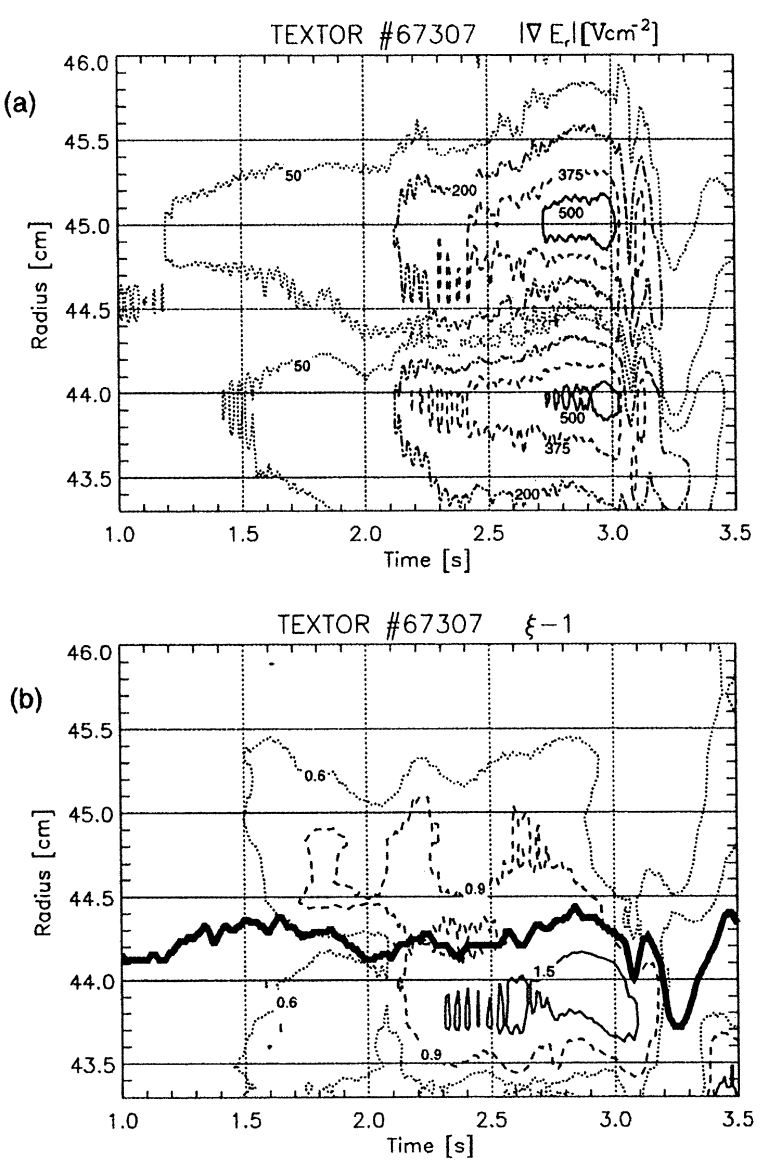

Fig. 6. (a) The gradient of the radial electric field and (b) the relative density gradient $\left(\xi-1=\left(\nabla n_{\text {bias }} / \nabla n_{\text {prebias }}\right)-\right.$ 1). The thick line in (b) shows the position of the maximal electric field strength $[a=r($ LCFS $) \simeq 45.5 \mathrm{~cm}]$.

FUSION SCIENCE AND TECHNOLOGY VOL. 47 FEB. 2005 
flows as is demonstrated by Fig. 5. The different curves represent different orientations of this Mach probe with respect to the magnetic field. Figure 3 shows that the current ratio changes at the precise location where the rotation caused by the electric field is situated. An extension $^{56}$ of the fluid model described in Ref. 53 permits to deduce the plasma rotation from the observed current ratio. Simultaneous measurement of the velocity components and the electric field at different radial locations then allowed us to benchmark this new algorithm to obtain the velocities from the current ratio by the constraint imposed by Eq. (1) (Refs. 56 and 57). The new technique was further checked by comparing it to the results obtained with Particle in Cell code simulations. ${ }^{58}$

To measure the electric field and the density with high spatial resolution a multipin rake probe (Fig. 1b) was used.

\section{THE EFFECT OF THE RADIAL ELECTRIC FIELD ON TRANSPORT AND CONFINEMENT}

With biasing, both the global particle and energy confinement times increase. It is generally observed that the particle confinement reacts stronger and that most of the energy increase is accountable by the density rise. ${ }^{39}$ Most effort has therefore been devoted to the study of particle transport. The major findings are listed as follows:

1. The improved particle confinement is the result of the creation of an edge barrier, located at the location

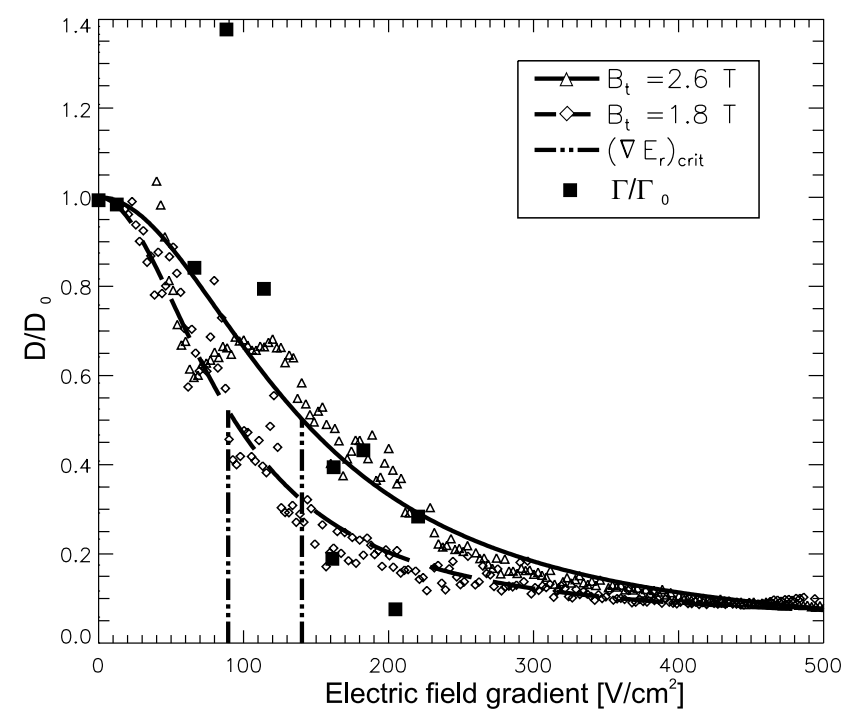

Fig. 7. Small symbols: variation of the local $D / D_{0}$ with $\nabla E_{r}$ at the location of maximum $\nabla E_{r}$ for two values of $B_{\phi, 0}$ (1.8 and 2.6 T) (Ref. 60). The solid curves are fits through the two data sets with the form of Eq. (3). The large rectangles are the results from fluctuation driven flux measurements at $B_{\phi, 0}=2.33 \mathrm{~T}$ (Ref. 61). of the maximum electric field gradient $\vec{\nabla} E_{r, \max }$. A barrier is defined here as the zone where the density gradient $\vec{\nabla} n$ steepens. Figure 6a (Ref. 59) shows a contour plot of $\left|\nabla E_{r}\right|$ measured in the edge region and is to be compared with Fig. 6b showing the corresponding contour data pertaining to the quantity $\xi-1$. Figure $6 \mathrm{~b}$ shows two maxima in the regions where $\left|\nabla E_{r}\right|$ or the corresponding rotation shear $\omega_{\vec{E} \times \vec{B}} \approx d V_{\vec{E} \times \vec{B}} / d r=B^{-1} \nabla E_{r}$ peaks and that the minimum in $\xi$ is closely related to the maximum value of $E_{r}$, indicated by the thick solid line in Fig. $6 \mathrm{~b}$. The plots clearly demonstrate that the radial transport is reduced (and thus the radial density gradient is enhanced) at the position where the field gradient is the most important. At this location the flow velocity created by the electric field's $\vec{E} \times \vec{B}$ drift is strongly sheared and quenches the turbulence, which is responsible for the loss of particles. ${ }^{59}$

2. A particle transport barrier can also be defined as a region of decreased diffusivity $D$. By means of the relations for the particle flux $\Gamma=-D \nabla n \propto H_{\alpha}$, the experimental measurements of the global recycling flux $H_{\alpha}$ and of the local $\nabla n$ yield a first approximation of the local value of $D$ at the barrier location. A typical experimental variation with $\nabla E_{r}$ of $D$, for two different magnetic fields, normalized to its zero-shear value $D_{o}$, is shown by the small symbols in Fig. 7. Also displayed in Fig. 7 is a fit by means of an analytical function,

$$
D / D_{0}=\left[D_{\text {res }}+D_{a n, 0}\left(1+\nabla E_{r}^{2} / \nabla E_{r, \text { crit }}^{2}\right)^{-1}\right] / D_{0},
$$

suggested by theory on nonlinear decorrelation ${ }^{22}$ by flow shear of turbulence characterized by a correlation time $\tau_{c o r r}$. Note that $D_{o}$ is assumed to consist of an anomalous part, responsive to shear, and a residual part $\left(D_{o}=D_{\text {res }}+\right.$ $\left.D_{a n, 0}\right)$, and that $\nabla E_{r, \text { crit }}=B \tau_{\text {corr }}^{-1}$ is the value of the field gradient at which the so-called Waltz's rule ${ }^{62}$ $\omega_{\vec{E} \times \vec{B}} \tau_{\text {corr }} \approx 1$ is satisfied. An independent experimental evaluation of the diffusivity was obtained from the measurement of the turbulent particle flux $\widetilde{\Gamma}$, found from the amplitudes and phases of the fluctuating density and poloidal electric field by means of a reciprocating probe $^{61,63,64}$ and the measured density profile $\left(D=\widetilde{\Gamma} \nabla n^{-1}\right)$ (Ref. 61). The result is shown by the large black symbols in Fig. 7. Both methods are seen to be remarkably consistent in demonstrating the role of shear and in the critical shear value that is needed to affect transport.

3. The causal role of $\nabla E_{r}$ in bringing about the transport changes has been further substantiated by showing that the field shear changes temporally lead the density gradient changes. ${ }^{33,65}$

4. As transport only reacts to the absolute field gradient, it should come as no surprise that the sign of the biasing polarity is not important in achieving improved confinement. It should, however, be noted that the combination of shear-related effects and direct effects 
of mobility can cause asymmetries with respect to polarity as reported in Ref. 35.

5. From the point of view of confinement, the observed bifurcation has no other effect but to allow a sudden increase from a low shear value, with weakly affected confinement, to a (much) higher shear and more strongly affected transport. The physical mechanism for $E_{r}$ bifurcation is different from that for confinement improvement. It is to be noted that confinement improvement already takes place before the bifurcation as the L-mode electric field already provides enough shear to affect the turbulence level.

\section{ACKNOWLEDGMENTS}

The many interesting results in this review have been obtained through the close and gratifying collaboration among the Ecole Royale Militaire/Koninklijke Militaire School, Forschungszentrum Jülich, and University of California, Los Angeles/University of California, San Diego teams working on TEXTOR.

\section{REFERENCES}

1. G. BUDKER, Plasma Physics and the Problem of Controlled Thermonuclear Research, Vol. 1, Pergamon Press, New York (1951).

2. J. GORMAN and L. H. RIETJENS, Phys. Fluids, 9, 2504 (1966).

3. T. STIX, Phys. Fluids, 14, 692 (1971).

4. E. STRAIT, Nucl. Fusion, 21, 943 (1981).

5. R. TAYLOR, Plasma Phys. Control. Thermonuclear Res., 3, 251 (1982).

6. W VII-A TEAM, Proc. 3rd Joint Varenna-Grenoble Int. Symp. Heating in Toroidal Plasma, Grenoble, France, Vol. 2 (1982).

7. F. WAGNER et al., Phys. Rev. Lett., 49, 1408 (1982).

8. R. TAYLOR et al., Phys. Rev. Lett., 63, 2365 (1989).

9. K. SHAING et al., Phys. Rev. Lett., 63, 2369 (1989).

10. K. BURRELL et al., Plasma Phys. Control. Fusion, 34, 1859 (1992).

11. E. STRAIT et al., Phys. Rev. Lett., 75, 4421 (1995).

12. L. LAO et al., Phys. Plasmas, 3, 1951 (1996).

13. E. MAZZUCATO et al., Phys. Rev. Lett., 77, 3145 (1996).

14. H. KIMURA et al., Phys. Plasmas, 3, 1945 (1996).

15. K. MIYAMOTO, Plasma Physics for Nuclear Fusion, The MIT Press, Cambridge, Massachusetts (1980).

16. B. KADOMTSEV and O. POGUTZE, Nucl. Fusion, 11, 67 (1971).

17. R. HAZELTINE, Phys. Fluids B, 1, 2031 (1989).

18. A. GALEEV and R. SAGDEEV, Sov. Phys. JETP, 26, 233 (1968).

19. T. STRINGER, Nucl. Fusion, 32, 1421 (1992).

20. H. MYNICK and W. HITCHON, Nucl. Fusion, 23, 1053 (1983).
21. W. VAN RIJN and S. HIRSHMAN, Phys. Fluids B, 1, 563 (1989).

22. K. ITOH, S.-I. ITOH, and A. FUKUYAMA, Transport and Structural Formation in Plasmas, Plasma Physics Series, IOP Publishing Ltd. (1999).

23. C. HORTON, Phys. Fluids, 12, 2132 (1969).

24. A. KAUFMAN, Phys. Fluids, 15, 1063 (1972).

25. R. HAZELTINE, Phys. Fluids, 24, 1164 (1981).

26. R. SHURYGIN, Plasma Phys. Reports, 21, 185 (1995).

27. R. HAZELTINE, Phys. Fluids, 17, 961 (1974).

28. B. LEHNERT, Phys. Fluids, 9, 1367 (1966).

29. H. BIGLARI et al., Phys. Fluids B, 2, 1 (1990).

30. Y. ZHANG and S. MAHAJAN, Phys. Fluids B, 4, 1385 (1992).

31. P. TERrY, Rev. Mod. Phys. B, 72, 109 (2000).

32. R. WEYNANTS and R. TAYLOR, Nucl. Fusion, 30, 945 (1990).

33. S. JACHMICH et al., Plasma Phys. Control. Fusion, 40, 1105 (1998).

34. S. JACHMICH et al., Czech. J. Phys., 48, S3 (1998).

35. R. WEYNANTS et al., Nucl. Fusion, 32, 837 (1992).

36. R. WEYNANTS et al., Proc. 17th Conf. Controlled Fusion and Plasma Heating, Amsterdam, The Netherlands, Vol. 1, European Physical Society (1990).

37. M. VAN SCHOOR et al., J. Nucl. Mater., 290-293, 962 (2000).

38. R. WEYNANTS et al., Proc. 20th EPS Conf. Controlled Fusion and Plasma Physics, Lisbon, Portugal, European Physical Society (1993).

39. R. WEYNANTS et al., Plasma Phys. Control Nucl. Fusion Res. 1990, 1, 473 (1991).

40. J. CORNELIS et al., Nucl. Fusion, 34, 171 (1994).

41. T. STRINGER, Nucl. Fusion, 33, 1249 (1993).

42. K. ITOH et al., Phys. Plasmas, 5, 4121 (1998).

43. J. HEIKKINEN et al., Phys. Rev. Lett., 84, 487 (2000).

44. J. HEIKKINEN et al., Phys. Plasmas, 8, 2824 (2001).

45. L. SPITZER, Jr., Physics of Fully Ionized Gases, 2nd ed., Interscience Publishers, New York (1962).

46. K. ITOH and S.-I. ITOH, Phys. Rev. Lett., 60, 2276 (1988).

47. K. ITOH and S.-I. ITOH, Nucl. Fusion, 32, 2243 (1992).

48. C. CHANG et al., Phys. Plasmas, 6, 1969 (1999).

49. S. GÜNTER et al., Proc. 18th Fusion Energy Conf., Sorrento, Italy, International Atomic Energy Agency (2000).

50. V. ROZHANSKY et al., Phys. Fluids B, 4, 1877 (1992).

51. P. LIEWER, Nucl. Fusion, 25, 543 (1985).

52. M. VAN SCHOOR et al., J. Nucl. Mater., 290-293, 963 (2001).

53. I. HUTCHINSON, Phys. Fluids, 30, 3777 (1987).

54. I. HUTCHINSON, Phys. Rev., 37, 4358 (1988).

55. I. HUTCHINSON, Phys. Fluids, 31, 2728 (1998).

56. H. VAN GOUBERGEN et al., Plasma Phys. Control. Fusion, 41, L17 (1999). 
57. H. VAN GOUBERGEN et al., Proc. 26th Conf. Controlled Fusion and Plasma Physics, Maastricht, The Netherlands, European Physical Society (1999).

58. J. GUNN, Czech. J. Phys., 48, S2 (1998).

59. S. JACHMICH et al., Proc. 24th Conf. Controlled Fusion and Plasma Heating, Berchtesgaden, Europhysics Conference Abstracts, Part IV, Vol. 21A, European Physical Society (1997).

60. S. JACHMICH et al., Czech. J. Phys., 49 S3, 191 (1999).
61. J. BOEDO et al., Proc. 18th Fusion Energy Conf., Sorrento, Italy, International Atomic Energy Agency (2000).

62. R. WALTZ et al., Phys. Plasmas, 1, 2229 (1994).

63. G. TYNAN et al., J. Nucl. Mater., 196-198, 770 (1992).

64. J. BOEDO et al., Nucl. Fusion, 42, 117 (2002).

65. S. JACHMICH et al., Proc. 29th Conf. Controlled Fusion and Plasma Heating, Montreux, Switzerland, Europhysics Conference Abstracts, European Physical Society (2002). 